\title{
PbS@P(NIPAM-co-TSt)功能复合物的合成及其荧光性能研究
}

\author{
王成志李润成浩姜韬殷明 \\ 陈欣 江金强* 刘晓亚* \\ (江南大学化学与材料工程学院 无锡 214122)
}

\begin{abstract}
摘要 以 4-氯亚甲基苯乙烯和硫脲为原料, 获得含有烷基异硫脲盐的苯乙烯类可聚合单体(TSt), 然后将其与 $N$-异丙基 丙烯酰胺(NIPAM) 共聚合合成温敏性聚合物 P(NIPAM-co-TSt), 进一步在弱碱性条件下使得有机异硫脲盐与 $\mathrm{Pb}(\mathrm{AcO})_{2} \cdot 3 \mathrm{H}_{2} \mathrm{O}$ 中的铅源反应生成含有 $\mathrm{PbS}$ 纳米粒子的 PbS@P(NIPAM-co-TSt)聚合物复合材料. 利用红外光谱(FTIR)、 核磁 $\left({ }^{1} \mathrm{H}\right.$ NMR)、X 射线衍射 $(\mathrm{XRD})$ 、苂光光谱 $(\mathrm{FL})$ 等手段对聚合物及复合物进行了各项表征，以确认 $\mathrm{PbS} @ \mathrm{P}(\mathrm{NIPAM}-\mathrm{co}-\mathrm{TSt}$ )复合物的合成, 并用纳米粒度仪和透射电镜法等对聚合物胶束及复合物颗粒的大小与形貌进行 了测定. 结果表明 PbS@P(NIPAM-co-TSt)纳米聚合物复合物既具有良好的荧光性能，且其荧光特征可随体系的温度和 浓度而发生变化、具有 LCST(低临界溶解温度)特征. 同时发现当聚合物体系中引入了 PbS 形成复合物后, 复合物的粒 径较之前聚合物的粒径分布明显减小. 我们认为类似的环境敏感型复合物能拓展聚合物的应用领域并在很多地方具有 广泛的应用前景.
\end{abstract}

关键词＼cjkstart烷基异硫脲盐; PNIPAM; 纳米粒子; 复合物; 温敏性

\section{Synthesis and Fluorescence Study of the Novel PbS@P(NIPAM-co-TSt) Complexes}

\author{
Wang, Chengzhi Li, Run Cheng, Hao Jiang, Tao Yin, Ming \\ Chen, Xin Jiang, Jinqiang* Liu, Xiaoya* \\ (School of Chemical and Material Engineering, Jiangnan University, Wuxi 214122)
}

\begin{abstract}
Nowadays, the environmental sensitive polymer complexes play a more and more important role in the field of science study. Herein we demonstrate that the alkylisothiouronium-containing styrene monomer (TSt) was synthesized via 4-vinylbenzyl chloride and thiourea, which could be copolymerized with NIPAM to synthesize the temperature sensitive polymer of P(NIPAM-co-TSt) by radical polymerization. Then, the PbS complexes of PbS@P(NIPAM-co-TSt) were prepared on the basis of the interaction between alkylisothiouronium and $\mathrm{Pb}(\mathrm{AcO})_{2} \cdot 3 \mathrm{H}_{2} \mathrm{O}$ in the aqueous solution of weakly alkaline. Its chemical structure was characterized by detail. For example, Fourier transformation infrared (FTIR), nuclear magnetic resonance $\left({ }^{1} \mathrm{H}\right.$ NMR), XRD (X-ray diffraction) and fluorescence spectrum to ensure the synthesis of PbS@P(NIPAM-co-TSt) complex. Besides, nanometer granularity apparatus, dynamic light scattering (DLS) and transmission electron microscope (TEM) were employed to survey the size and physiognomy of polymer micelles and complexes. The photoluminescence properties were also studied. It was found that PbS@P(NIPAM-co-TSt) complex exhibits characteristic fluorescence intensity, which was promoted by the PbS nanoparticles. Meanwhile, its fluorescence intensity increased along with the temperature, which very consists with the LCST (lower critical solution temperature) characteristic of PNIPAM. The results indicated that its fluorescene intensity would go up with the addition of the complex's concentration and the raising of the temperature. The diameter of PbS@P(NIPAM-co-TSt) complex can also be reduced obviously by introducing the PbS nanoparticles to the system. What is more, its diameter had a declining trend when the temperature exceeded the LCST of it and kept stable in the end. Thus, on the one hand, these kinds of complexes possess the properties in both polymers and nanoparticles, such as $\mathrm{PbS}$. On the other hand, we think that such environmental sensitive complexes must have a bright future, and also could make a lot of potential applications as optical materials in a great many areas, such as biological, medical science, cells, chemistry and so on.

Keywords alkylisothiouronium; PNIPAM; nanoparticles; complexes; temperature sensitive
\end{abstract}

\footnotetext{
*E-mail: jiangjq@jiangnan.edu.cn; 1xy@jiangnan.edu.cn; wcz_19880716@126.com

Received March 20, 2012; published June 11, 2012.

Supporting information for this article is available free of charge via the Internet at http://sioc-journal.cn.

Project supported by the National Natural Science Foundation of China (Nos. 20704017, 50973044) and the Program for New Century Excellent Talents in University (NCET-10-0452).

项目受国家自然科学基金(Nos. 20704017, 50973044)和 2010 年度新世纪优秀人才支持计划(NCET-10-0452)资助.
} 


\section{1 引言}

自 1990 年以来, 由于纳米粒子(nanoparticles, NPs) 及其聚合物纳米复合物 (polymer nanocomposites, PNCs) 在光学、发光学、电学以及催化等基础研究与应用研究 领域得到了极大的发展 ${ }^{[1 \sim 7]}$, 同时在材料科学领域, 随 着对低维度尺寸材料日趋成熟的操控和制备, 人们对杂 化复合材料体系越来越感兴趣. 由于聚合物在日常生 活、航空航天、能源、通信科技等方面的广泛应用, 社 会对高分子材料性能的要求越来越高. 同时由于人们的 需求日趋多样化, 因此单一的均聚物、共聚物和共混物 已经难以满足不同形式的需求, 更为重要的是依据纳米 粒子在科学和应用上的重要性 ${ }^{[8]}, \mathrm{NPs} @$ 聚合物复合材 料显得尤为重要, 是材料科学发展中一类新兴的功能材 料. 目前, 人们已成功地利用各种方法制备出 $\mathrm{ZnS}, \mathrm{CdS}$, $\mathrm{CdSe}, \mathrm{Fe}_{2} \mathrm{O}_{3}, \mathrm{SiO}_{2}, \mathrm{CdSe}$, CdTe 等 NPs, 并对它们的应用 进行了广泛而深入的研究. 在目前的主要研究领域内, 根据 NPs 的不同可以大致分为高分子与单一 NPs 的直 接复合 ${ }^{[9,10]}$ 以及高分子与核壳型纳米粒子的复合两 种 ${ }^{[11,12]}$. 同时，根据高分子的种类不同，例如聚合物的 不同响应性, 出现了很多新型的复合材料, 例如将具有 $\mathrm{pH}$ 值响应性 ${ }^{[13]}$ 的 PAA 与 NPs 复合等 ${ }^{[14 ~ 16]}$; 将具有温度 响应性 ${ }^{[17,18]}$ 的 PNIPAM 与 NPs 复合 ${ }^{[19 \sim 23]}$; 同时实现温 度响应性和顺磁性的 NPs 复合材料. 这些复合材料能同 时保留化学响应性和 NPs 的杰出性能, 为实现特殊领域 的应用开辟了新的思路.

本文以 4-氯亚甲基苯乙烯和硫腿为原料, 获得含有 烷基异硫脲盐的苯乙烯类可聚合单体(TSt), 然后将其 与 $N$-异丙基丙烯酰胺(NIPAM)共聚合合成温敏性聚合 物 P(NIPAM-co-TSt), 进一步在弱碱性条件下使得有机 异硫脲盐与 $\mathrm{Pb}(\mathrm{AcO})_{2} \cdot 3 \mathrm{H}_{2} \mathrm{O}$ 中的铅源反应生成含有 $\mathrm{PbS}$ 纳米粒子的 PbS@P(NIPAM-co-TSt) 聚合物复合材料. 此法可以将聚合物的优异性能(多环境响应性等)赋予纳 米粒子, 并对纳米粒子的性能产生影响, 不仅提高了纳 米粒子的均匀度和分散性, 同时还改善了纳米粒子的光 学性能, 从而获得性能优异的纳米粒子@聚合物复合 物——PbS@P(NIPAM-co-TSt).

\section{2 结果与讨论}

\subsection{PbS@P(NIPAM-co-TSt)的设计与合成}

聚 $N$-异丙基丙烯酰胺(PNIPAM)是一种应用十分广 泛的温敏性聚合物, 它同时包含有亲水性的酰胺键和疏 水性的异丙基基团. 正是由于这种相互竞争作用的存在 使得 PNIPAM 具有低温亲水、高温疏水的独特性质. 当 温度小于其 LCST 时, 亲水性的酰胺键能与水分子形成 氢键并占据主导地位, 氢键的存在使聚合物能够溶于 水; 当温度大于 LCST 时, 氢键作用得到减弱, 疏水作 用相对增强并开始占据主导地位, 故聚合物不溶于水. 这种独特的亲/疏水性转变行为引起了人们的极大兴趣,
也开始了 PNIPAM 类聚合物在环境响应性材料领域的 应用 ${ }^{[24]}$.

$\mathrm{PbS}$ 是一种重要的直接带隙的半导体材料, 是一种 研究量子尺寸效应的典型材料, 它的量子束缚效应程度 是其它半导体材料的数倍. 我们先通过合成一种含被保 护颈基的苯乙烯单体而后经过聚合将颈基接到聚合物 链上形成颈基功能化聚合物，由于統基具有很强的配位 能力，因此我们之后在碱性条件下将颈基释放出来并在 体系中引入醋酸铅即可制备得到性能优异的纳米粒子 $@$ 聚合物复合物—-PbS@P(NIPAM-co-TSt).

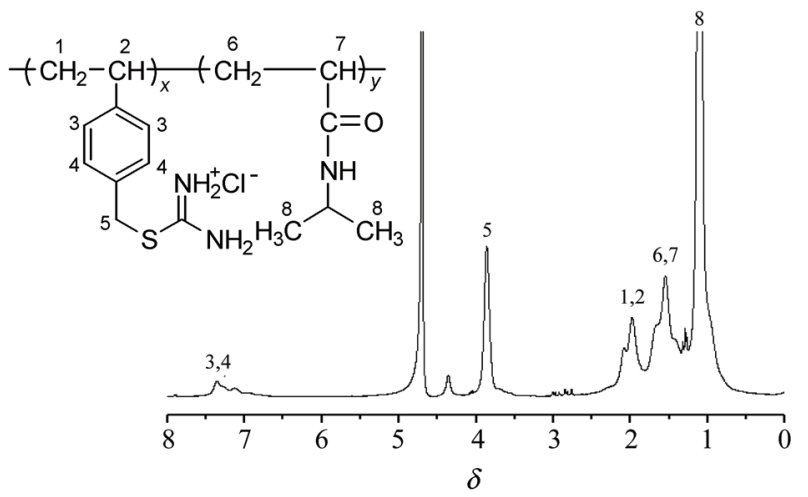

图 1 P(NIPAM-co-TSt)的核磁谱图

Figure $1{ }^{1} \mathrm{H}$ NMR spectrum of P(NIPAM-co-TSt)

图 1 为聚合物 $\mathrm{P}(\mathrm{NIPAM}-\mathrm{co}-\mathrm{TSt})$ 的 ${ }^{1} \mathrm{H}$ NMR 谱图, $\delta$ 7.35 附近处为苯环上的质子峰; $\delta 4.70$ 附近处为溶剂重 水的质子峰; $\delta 3.86$ 附近处为与颈基相连的亚甲基 $\left(\mathrm{CH}_{2}\right)$ 质子峰; $\delta 2.09$ 和 $\delta 1.55$ 附近处为聚合物分子链上的质 子峰; $\delta 1.12$ 处为与胺基相连的甲基 $\left(\mathrm{CH}_{3}\right)$ 质子峰, 经过 积分可知 TSt 与 NIPAM 比例为 $1: 9.2$, 与投料比相似. 核磁图说明成功地合成了聚合物 P(NIPAM-co-TSt).

\section{$2.2 \mathrm{NPs} @$ 聚合物复合物荧光性能的探讨}

\subsection{1 浓度对复合物荧光性能的影响}

荧光性能是 $\mathrm{PbS}$ 纳米粒子的一个重要光学特性, 复 合材料是否具有荧光性能是聚合物对纳米粒子实现包 裹的重要体现. 根据上面提到合成方法, 我们制备了 5 组不同浓度的 PbS@P(NIPAM-co-TSt)复合物溶液, 浓 度分别为 $0.6,0.75,1,1.5,3 \mathrm{mg} / \mathrm{mL}$ 并分别进行了荧光测 量, 观察浓度变化时其苂光性能的变化, 结果如图 2 所 示.

由图 2 可知, PbS@P(NIPAM-co-TSt)的苂光强度随 着浓度含量的增加呈现出逐渐增大后减小的趋势, 这是 因为当 $\mathrm{PbS} @ \mathrm{P}(\mathrm{NIPAM}-\mathrm{co}-\mathrm{TSt})$ 浓度增加时使得 $\mathrm{PbS}$ 纳 米粒子的含量增多, 由于纳米粒子的荧光强度具有几何 累加性，同时随着复合物的的浓度变大，纳米粒子@聚 合物之间的距离大大缩短, 纳米粒子之间存在强的相互 作用，这种相互作用使体系的荧光强度大大的增强. 但 是随着 $\mathrm{PbS}$ 含量的提高, 复合物中聚合物与 $\mathrm{PbS}$ 之间的 作用增强，导致非辐射能量转移，因此荧光发射减 


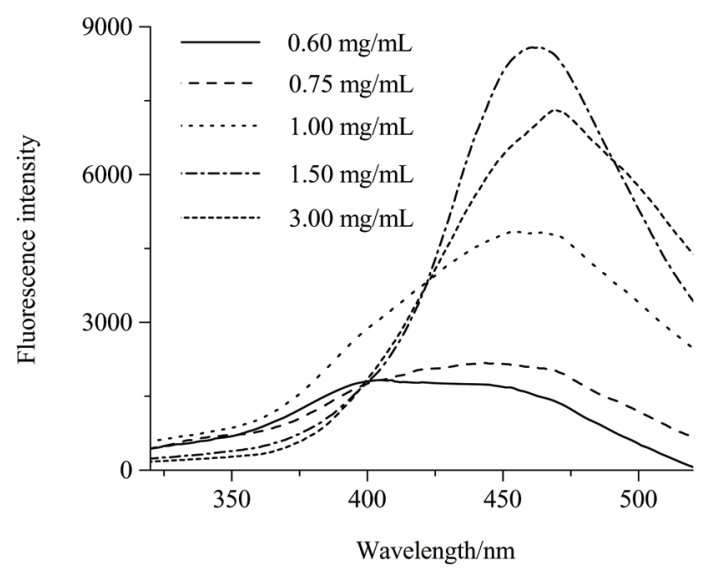

图 2 不同 PbS@P(NIPAM-co-TSt)浓度时 NPs@聚合物复合材料的荧 光强度变化(其中激发波长为 $280 \mathrm{~nm}$ )

Figure 2 FL spectra of PbS@P(NIPAM-co-TSt) hybrid materials taken at different concentrations of PbS@P(NIPAM-co-TSt). The excitation wavelength was $280 \mathrm{~nm}$

弱 ${ }^{[25 ~ 27]}$. 此外, 我们发现 5 组样品荧光峰出现了一定的 红移, 其主要原因可能是 $\mathrm{PbS}$ 纳米粒子因聚集而使复合 物粒径增大导致的.

\subsection{2 温度对 PbS@P(NIPAM-co-TSt)荧光强度的影响}

我们对复合材料温敏性也进行了考察，主要考察了 温度变化对于复合材料的荧光性能的影响, 经过紫外透 光率的测试, 可以看到当温度升高到 $33{ }^{\circ} \mathrm{C}$ 左右时, 聚 合物的透光率开始发生突变, 因此我们可以认为聚合物 的 LSCT 大约为 $33{ }^{\circ} \mathrm{C}$ 左右, 结果如图 3 所示.

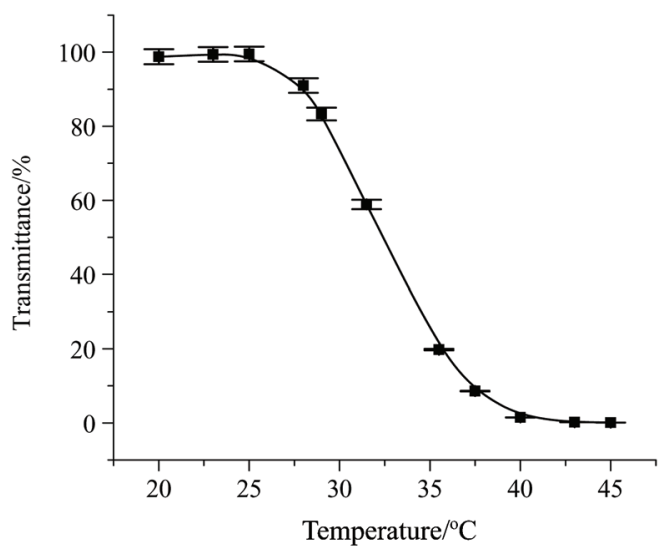

图 3 聚合物 P(NIPAM-co-TSt)的透光率与温度变化关系

Figure 3 The UV transmittance of P(NIPAM-co-TSt) at different temperatures

根据以上测试结果, 我们对于复合物的温敏性荧光 测试所选定温度测试区间为 $25 \sim 55{ }^{\circ} \mathrm{C}$, 结果如图 4 所示.

由图 4 可知, 在不同的温度条件下, 复合材料的最 大吸收峰都出现在 $468 \mathrm{~nm}$ 附近处, 但温度越高其荧光 强度越高. 为了直观地看出温度对于荧光强度的影响, 我们将不同温度下的最大吸收峰强度相对于初始吸收 峰数值的变化幅度对温度作图, 如图 5 所示, $\Delta F$ 为其他 温度时苂光吸收强度相对于初始 $25{ }^{\circ} \mathrm{C}$ 时的变化量, $F_{0}$

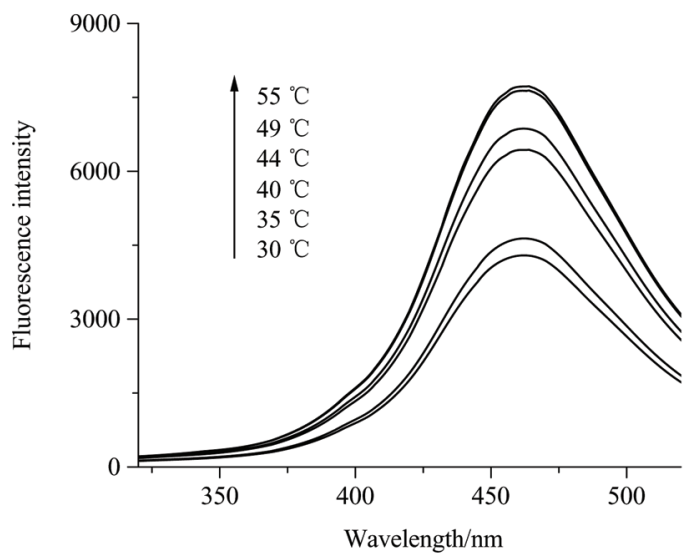

图 $4 \mathrm{PbS} @ \mathrm{P}(\mathrm{NIPAM}-\mathrm{co}-\mathrm{TSt})$ 复合材料温敏的荧光谱图(温度变化区 间为 $25 \sim 55{ }^{\circ} \mathrm{C}$, 激发波长为 $280 \mathrm{~nm}$ )

Figure 4 FL spectra of PbS@P(NIPAM-co-TSt) hybrid materials taken at different temperatures between $25{ }^{\circ} \mathrm{C}$ and $55{ }^{\circ} \mathrm{C}$. The excitation wavelength was $280 \mathrm{~nm}$

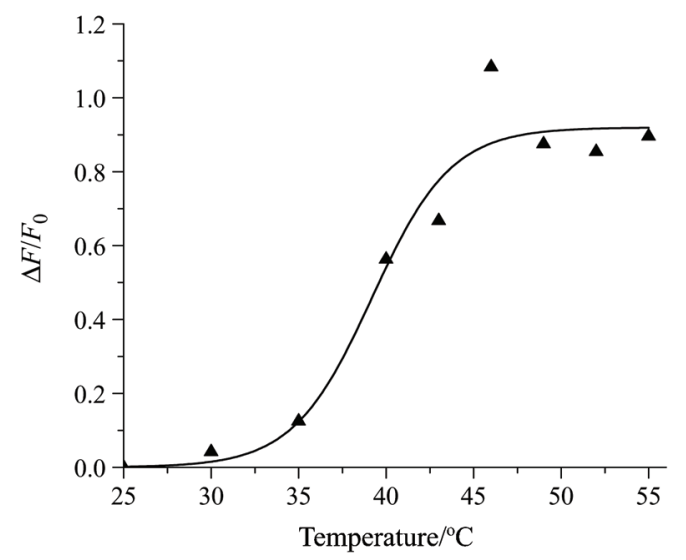

图 5 复合材料的荧光发射峰强度随温度的变化关系趋势

Figure 5 Temperature dependence of the fluorescence emission intensity

为 $25{ }^{\circ} \mathrm{C}$ 下的苂光强度.

通过图 5, 我们可以直观地看出, 当温度升高时, 复合物的荧光强度开始的时候变化很小, 当达到复合物 的 LCST 附近时, 其苂光强度开始急剧地增强，而后逐 渐达到最大值, 并逐渐趋于稳定. 这主要是因为当温度 小于 LCST 时, $\mathrm{PbS}$ 纳米粒子以个体状态的形式分散在 溶液中, 它们之间的距离较远因而相互作用较弱, 从而 苂光强度随温度变化不大; 当温度升高到 LCST 附近时, NPs 开始聚集在一起, 它们彼此接近相互作用，从而荧 光强度急剧地增加直至稳定.

\section{$2.3 \mathrm{PbS} @ \mathrm{P}(\mathrm{NIPAM}-\mathrm{co}-\mathrm{TSt}$ )复合物颗粒大小的探讨}

2.3.1 聚合物粒径与 PbS@P(NIPAM-co-TSt)复合物粒 径的比较

为了比较纳米粒子接入前后对聚合物颗粒尺寸的 变化影响, 我们对聚合物以及纳米粒子@聚合物复合物 颗粒的大小进行了粒径测试, 结果如图 6 所示. 


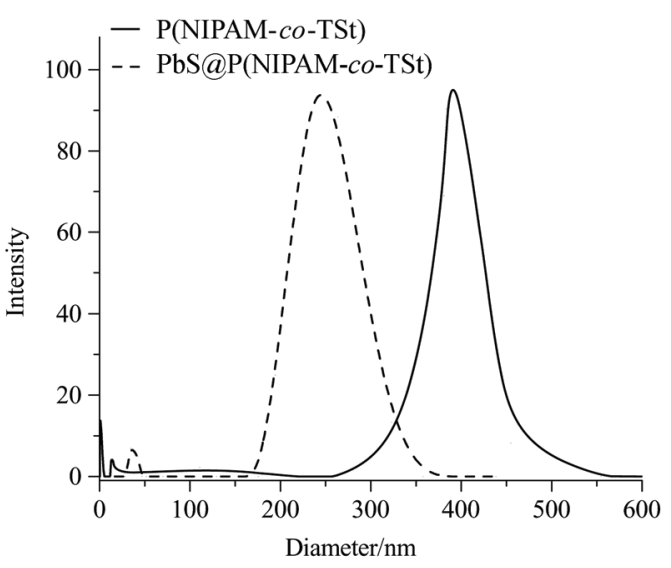

图 $6 \mathrm{P}(\mathrm{NIPAM}-c o-\mathrm{TSt})$ 胶束与 $\mathrm{PbS} @ \mathrm{P}(\mathrm{NIPAM}-c o-\mathrm{TSt})$ 颗粒的尺寸分 布图

Figure 6 The diagram of size distribution of P(NIPAM-co-TSt) micelles and PbS@P(NIPAM-co-TSt) particles

从图中我们可以看到, P(NIPAM-co-TSt)胶束的粒 径分布约为 $400 \mathrm{~nm}$ 左右; 而 PbS@P(NIPAM-co-TSt)复 合物的粒径分布约为 $250 \mathrm{~nm}$ 左右. 因此, NPs 的引入明 显地减小了聚合物的粒径, 这主要是因为在常温条件 下, NIPAM 具有很好的亲水性, 故聚合物链趋向于伸展 状态. 当在体系中引入 NPs 后, NPs 的存在使其对聚合 物链有一定的牵扯作用, 从而聚合物链伸展状态受到抑 制使 NPs@聚合物的颗粒尺寸明显减小.

\subsubsection{PbS@P(NIPAM- $c o-\mathrm{TSt}$ ) 复合物颗粒的温敏性探讨}

同样，基于聚合物的温敏性特点，我们也对复合物 的温度对粒径的影响进行了测试, 为了直观地表现这种 变化, 我们将粒径对温度作图, 从图 7 中我们可以清楚 地看到, 当温度升高时, PbS@P(NIPAM-co-TSt)颗粒的 粒径不断减小. 但减小的幅度随温度的变化而有所不 同, 当温度小于 LCST 时, 减小的幅度不大; 当温度邻 近 LCST 时, 复合物的粒径分布开始迅速减小; 当温度 超过 LCST 时, 粒径分布缓慢变化并趋向于稳定. 这是 因为体系中同时存在亲水的相互作用和疏水的相互作 用, 当温度较低时, 亲水的相互作用占据优势, 颗粒较 分散得到的颗粒尺寸较大; 当温度升高时, 疏水间的相 互作用得到增强, 颗粒开始聚集而呈现出较小的有效粒 径. 这也是 PbS@P(NIPAM-co-TSt)复合物温敏性的体现.

\section{$2.4 \mathrm{PbS} @ \mathrm{P}(\mathrm{NIPAM}-\mathrm{Co}-\mathrm{TSt})$ 复合物的形貌}

根据粒径的测试结果, 我们将 PbS@P(NIPAM-co$\mathrm{TSt}$ )进行了 TEM 测试, 以确定复合物的大小以及形貌, 可以得出复合物的粒径大小在 $200 \mathrm{~nm}$ 附近, 这与之前 的粒径测试结果相一致, 如图 8 所示.

我们从 TEM 图中可以看到, 在聚合物胶束的内部 分布着许多的小黑点, 我们认为由于 PbS NPs 的尺寸在 $10 \mathrm{~nm}$ 范围内, 因此认为这就是 PbS NPs 的存在, 而外 层颜色较浅的包裹层即为聚合物层. 聚合物包覆在纳米 粒子的外围为纳米粒子提供了稳定性存在的依据, 同时

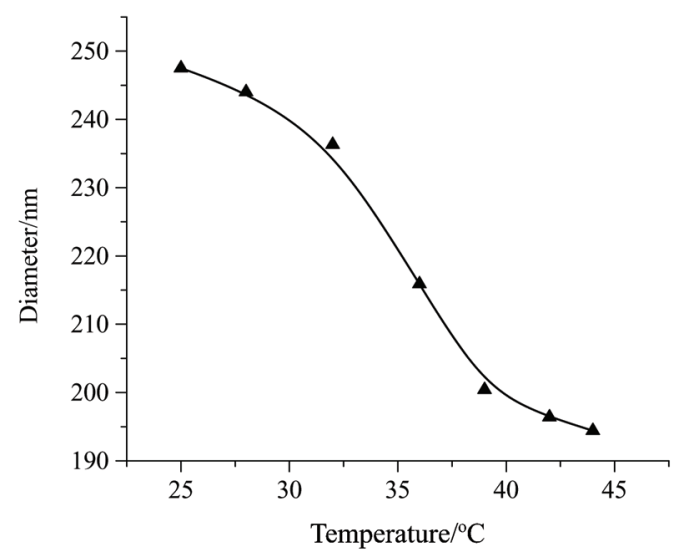

图 $7 \mathrm{PbS} @ \mathrm{P}(\mathrm{NIPAM}-\mathrm{co}-\mathrm{TSt})$ 复合物的粒径分布随温度的变化 Figure 7 The temperature dependence of diameter $(\mathrm{nm})$ of PbS@P(NIPAM-co-TSt) particles

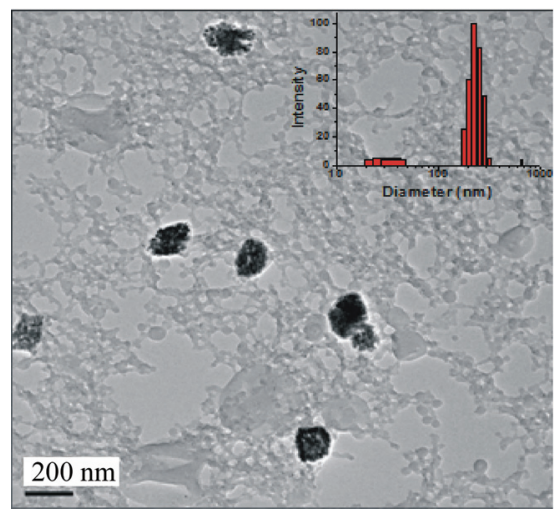

图 8 PbS@P(NIPAM-co-TSt)的 TEM 图

Figure 8 TEM image of PbS@P(NIPAM-co-TSt)

NPs 也为聚合物提供了依附的中心, 整个复合物的颗粒 大小是由 PbS NPs 和 P(NIPAM-co-TSt) 聚合物共同决定的.

\section{3 结论}

在制备了 $N$-异丙基丙烯酰胺-統基功能化苯乙烯二 元无规共聚物后, 体系中引入 $\mathrm{PbS}$ 纳米粒子与聚合物复 合，制备合成了具有温敏性的 PbS@P(NIPAM-co-TSt) 复合物, 并且在合成步骤中避免了常规合成方法中 NPs 易被氧化的问题，合成过程简便效率高，制备的复合物 稳定性好并且光学性能优良. 之后对合成的 $\mathrm{PbS} @ \mathrm{P}(\mathrm{NIPAM}-\mathrm{co}-\mathrm{TSt})$ 复合物采用苂光(FL)、粒径分析, 并探讨了浓度、温度等变化时荧光强度和粒径的变化, 证明了合成的复合物具有较为优异的荧光及温敏性能.

\section{4 实验部分}

\subsection{NPs@聚合物复合材料 PbS@P(NIPAM-co-TSt) 的合成}

4.1.1 温敏性聚合物 P(NIPAM-co-TSt)的合成

在 $150 \mathrm{~mL}$ 的圆底烧瓶中加入 $15.262 \mathrm{~g}$ 对氯甲基苯 乙烯 $(0.1 \mathrm{~mol}), 8.3732 \mathrm{~g}$ 硫脲 $(0.11 \mathrm{~mol})$, 两者 $1: 1$ 反应, 
硫嫝略微过量. 加入乙醇(作为溶剂) $50 \mathrm{~mL}$ 和对苯二酚 (作为阻聚剂, 其加入量为质量分数保持在 $0.1 \%$ 左右). 加入搅拌子于 $90{ }^{\circ} \mathrm{C}$ 条件下冷凝回流进行反应, 反应时 间大概持续 $7 \mathrm{~h}$ 后用乙醇重结晶得到含有被保护颈基的 苯乙烯单体(TSt). 之后在 $150 \mathrm{~mL}$ 圆底烧瓶中加入放基 受保护的苯乙烯类单体 (TSt) $0.4576 \mathrm{~g}\left(2 \times 10^{-3} \mathrm{~mol}\right) 、 N$ 异丙基丙烯酰胺(NIPAM) $2.0340 \mathrm{~g}\left(1.8 \times 10^{-2} \mathrm{~mol}\right)$ 、引 发剂偶氮二异丁腈(AIBN) $0.0544 \mathrm{~g} 、 \mathrm{DMF}$ (溶剂) $20 \mathrm{~mL}$, 其中 NIPAM 与 TSt 物质的量比为 $9: 1$. 加入搅拌子后 抽真空(大概持续 5 10 min), 使反应在 70 $80{ }^{\circ} \mathrm{C}$ 的油 浴锅中进行, 反应时间为 $24 \mathrm{~h}$. 将产物透析得到温敏性 二元共聚物 P(NIPAM-co-TSt), $M_{\mathrm{n}}=7861 \mathrm{~g} / \mathrm{mol}$.<smiles>C=Cc1ccc(CCl)cc1</smiles>

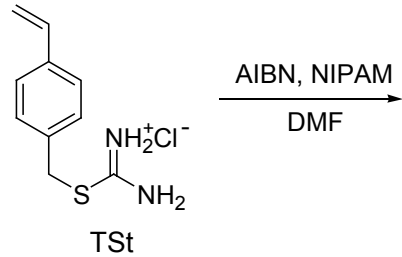<smiles>CCCc1ccc(CSC(N)=[N+]=[Cl+])cc1</smiles>

P(NIPAM-Co-TSt)

图式 1 P(NIPAM-co-TSt)的合成

Scheme 1 Synthetic route to the P(NIPAM-co-TSt)

${ }^{1} \mathrm{H}$ NMR (500 MHz, $\left.d-\mathrm{H}_{2} \mathrm{O}\right) \delta$ (TMS): $7.43(2 \mathrm{H}$, $\mathrm{ArH}), 7.15$ (2H, ArH), 3.9 (2H, Ar- $\left.\mathrm{CH}_{2}-\mathrm{S}\right), 1.1\left(6 \mathrm{H}, \mathrm{CH}_{3}\right)$, $1.5 \sim 2.0(3 \mathrm{H}, \mathrm{H}$ on the polymer chain).

\subsubsection{PbS@P(NIPAM-co-TSt)的合成}

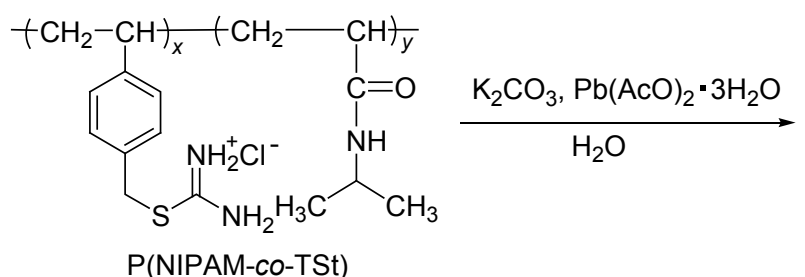<smiles>CCCCCCCCCCCCCCSP(=O)(O)SCc1ccc(CCC)cc1</smiles>

PbS@P(NIPAM-Co-TSt)

图式 2 PbS@P(NIPAM-co-TSt)的合成 Scheme 2 Synthetic route to the PbS@P(NIPAM-co-TSt)
取二元共聚物 $0.47 \mathrm{~g}$ (颈基含量为 $6 \times 10^{-3} \mathrm{mmol}$ )置 于 $150 \mathrm{~mL}$ 圆底烧瓶中, 三水合醋酸铅 $4.8 \mathrm{mg}(1.2 \times$ $10^{-2} \mathrm{mmol}$, 过量)和碳酸钾 $1.8 \mathrm{mg}\left(1.2 \times 10^{-2} \mathrm{mmol}\right)$, 加 入一定量的超纯水(超纯水的投入量直接关系到溶液中 复合物的浓度，如 $50 \mathrm{~mL}$ 超纯水时可认为复合物的浓度 为 $6.00 \mathrm{mg} / \mathrm{mL}$ ), 将固体物质混合溶解, 溶解后的溶液 在 $80{ }^{\circ} \mathrm{C}$ 的油浴锅中反应, 反应时间为 $12 \mathrm{~h}$, 透析后得 到产物 PbS@P(NIPAM-co-TSt).

\section{References}

[1] Priester, C.; Lannoo, M. Curr. Opin. Solid State Mater. Sci. 1997, 2, 716.

[2] Nikodem, T.; Dominik, J.; Han, M. Y.; Julius, V. G. Prog. Polym. Sci. 2009, 34, 393.

[3] Poznyak, S. K.; Talapin, D. V.; Shevchenko, E. V.; Weller, H. Nano Lett. 2004, 4, 693.

[4] Michael, T. Ann. Phys. 2004, 13, 5.

[5] Zhang, B. B.; Xing, D.; Lin, C.; Guo, F. F.; Zhao, P.; Wen, X. J.; Bao, Z. H.; Shi, D. L. J. Nanopart. Res. 2011, 13, 2407.

[6] Lee, D. K.; Lee, Y.-k. Macromol. Res. 2010, 10, 1007.

[7] Chen, Y.; Rosenzweig, Z. Anal. Chem. 2002, 74, 5132

[8] Koh, W.-k.; Saudari, S. R.; Fafarman, A. T.; Kagan, C. R.; Murray, C. B. Nano Lett. 2011, 11, 4764 .

[9] Wang, C.-W.; Oskooei, A.; Sinton, D.; Moffitt, M. G. Langmuir 2010, 26, 716.

[10] Wang, M. F.; Felorzabihi, N.; Guerin, G.; Haley, J. C.; Scholes, G. D.; Winnik, M. A. Macromolecules 2007, 40, 6377.

[11] Wang, M. F.; Zhang, M.; Qian, J. S.; Zhao, F.; Shen, L.; Scholes, G. D.; Winnik, M. A. Langmuir 2009, 25, 11732.

[12] Yu, X. Y.; Liao, J. Y.; Qiu, K. Q.; Kuang, D. B.; Su, C. Y. Acs Nano 2010, 10, 1021

[13] Zhu, L. J.; Shi, Y. F.; Tu, C. L.; Wang, R. B.; Pang, Y.; Qiu, F.; Zhu, X. Y.; Yan, D. Y.; He, L.; Jin, C. Y.; Zhu, B. S. Langmuir 2010, 26 8875

[14] Yang, Y.-C.; Lu, H.-H.; Wang, W.-T.; Liau, I. Anal. Chem. 2011, 83, 8267.

[15] Yusuf, H.; Kim, W.-G.; Lee, D. H.; Guo, Y. Y.; Moffitt, M. G. Langmuir 2007, 23, 868.

[16] Wang, C.-W.; Oskooei, A.; Sinton, D.; Moffitt, M. G. Langmuir 2010, 26, 716 .

[17] Agrawal, M.; Rubio-Retama, J.; Zafeiropoulos, N. E.; Gaponik, N.; Gupta, S.; Cimrova, V.; Lesnyak, V.; Lopez-Cabarcos, E.; Tzavalas, S.; Rojas-Reyna, R.; Eychmuller, A.; Stamm, M. Langmuir 2008, 24, 9820 .

[18] Hou, Y.; Ye, J.; Gui, Z.; Zhang, G. Z. Langmuir 2008, 24, 9682.

[19] Janczewski, D.; Tomczak, N.; Han, M.-Y. Macromolecules 2009, 42, 1801.

[20] Guo, J.; Yang, W. L.; Wang, C. C.; He, J.; Chen, J. Y. Chem. Mater. 2006, 18,5554 .

[21] Ye, J.; Hou, Y.; Zhang, G. Z.; Wu, C. Langmuir 2008, 24, 2727.

[22] Ye, F. M.; Wu, C. F.; Jin, Y. H.; Chan, Y.-H.; Zhang, X. J.; Chiu, D. T. J. Am. Chem. Soc. 2011, 133, 8146.

[23] Lao, U. L.; Mulchandani, A.; Chen, W. J. Am. Chem. Soc. 2006, 128, 14756.

[24] Shu, Q. Z.; Chen, X.; Chen, K. H.; Jiang, J. Q.; Ni, Z. B.; Zhang, H. W.; Liu, X. Y.; Chen, M. Q. Acta Phys.-Chim. Sin. 2010, 26, 2845. (舒巧珍，陈欣，陈开花，江金强，倪忠斌，张红武，刘晓亚，陈明 清, 物理化学学报, 2010, 26, 2845.)

[25] Carrot, G.; Scholz, S. M.; Plummer, C. J. G.; Hilborn, J. G.; Hedrick, J. L. Chem. Mater. 1999, 11, 3571.

[26] Nair, P. S.; Radhakrishnan, T.; Revaprasadu, N.; Kolawole, G. A.; O'Brien, P. Chem. Commun. 2002, (6), 564.

[27] Dong, Y. M.; Lu, J. M.; Xu, Q. F. J. Macromol. Sci. 2008, 45, 37.

(Cheng, B.; Fan, Y.) 